\title{
Domain Decomposition Method for Diffuse Optical Tomography
}

\author{
Kiwoon Kwon ${ }^{a}$, Il-young Son ${ }^{a}$, and Birsen Yazici ${ }^{a}$ \\ ${ }^{a}$ Electrical, Computer, and System Engineering, Rensselaer Polytechnic Institute
}

\begin{abstract}
Diffuse optical tomography is modelled as an optimization problem to find the absorption and scattering coefficients that minimize the error between the measured photon density function and the approximated one computed using the coefficients. The problem is composed of two steps: the forward solver to compute the photon density function and its Jacobian (with respect to the coefficients), and the inverse solver to update the coefficients based on the photon density function and its Jacobian attained in the forward solver. The resulting problem is nonlinear and highly ill-posed. Thus, it requires large amount of computation for high quality image. As such, for real time application, it is highly desirable to reduce the amount of computation needed. In this paper, domain decomposition method is adopted to decrease the computation complexity of the problem. Two level multiplicative overlapping domain decomposition method is used to compute the photon density function and its Jacobian at the inner loop and extended to compute the estimated changes in the coefficients in the outer loop. Local convergence for the two-level space decomposition for the outer loop is shown for the case when the variance of the coefficients is small.
\end{abstract}

\section{INTRODUCTION}

Diffuse optical tomography (DOT) is a promising medical imaging modality using near infra-red spectroscopy to ascertain the optical properties of human tissues. It is a noninvasive and inexpensive alternative to other imaging modalities such as MRI. DOT also provides better functional information than MRI making it suitable for complementary use in conjunction with other imaging modalities that may provide better spatial resolution. ${ }^{1-3}$

The turbid nature of biological tissue makes inverse DOT a nonlinear and ill-posed problem. Hence, it is a computationally challenging problem that calls for the use of nonlinear minimization methods with regularization to stabilize the solution.

A popular way of modelling photon propagation in highly scattering media is the frequencydomain photon diffusion equation derived from a simplifying assumptions applied to photon transport equation. Based on this forward model, absorption and scattering coefficients are reconstructed to minimize the error between the measured photon density and the computed photon density.

We now present an overview of our problem formation and the methods used in its solution. The overall problem is posed as an optimization problem. It is formulated using two coupled steps, aptly named forward and inverse. Each consists of an iterative solver whose solutions are used as an input to the other solver. More precisely, the forward solver computes the photon density and its Jacobian with respect to the optical coefficients, and the inverse solver updates the optical coefficients based on the output of the forward step. The updated coefficients are then used in the forward solver to recompute the photon density and its Jacobian and so on.

The computational complexity of DOT reconstruction quickly grows with number of pixels and dimensions. Thus, real time computation of DOT needs further numerical techniques to simplify the complexity of the problem. We suggest the use of domain decomposition methods to tackle this problem. Domain decomposition methods have previously been studied in the area of numerical

Send correspondence to Birsen Yazici: E-mail: yazici@ecse.rpi.edu 
solution to partial differential equations over the last two decades, motivated by the need for fast and efficient algorithm for solving large-scale, three-dimensional problem. One advantage of domain decomposition method is that it allows for distributed parallel numerical solvers on smaller subdomains, making the computation extremely efficient.

In this paper, we explore the classic Schwarz method, developed largely for solving elliptic problems. The Schwarz method comes in two flavors, additive and multiplicative. ${ }^{4-9}$ We chose to adopt the two-level multiplicative Schwarz method as described in ${ }^{10}$ to the forward problem. It is known that multiplicative Schwarz method converges faster than additive Schwarz method. ${ }^{11,12}$

Since the photon density function for a given source is shaped like a Green's function that is rapidly varying around the source, the contribution from the source in the subdomains far from it are neglected. As described in ${ }^{10}$ the two-level multiplicative Schwarz method uses coarse grid correction applied to the entire domain after performing fine grid solution at each subdomain, accounting for low frequency errors that are not handled well at the fine grid level, resulting in computational efficiency.

The main focus of this paper is to apply the idea of two-level Domain decomposition to the inverse solver minimizing optical coefficients. Badea and Tai et al. ${ }^{13-20}$ have previously applied domain decomposition techniques to a constrained convex minimization problem coming from variational inequalities using space decomposition method. It has been shown that multigrid can be viewed as a special case of such space decomposition. ${ }^{21}$ We apply this space decomposition idea to DOT imaging. The present algorithm for inverse solver is as follows. First, we divide the whole domain into disjoint subdomains considering finite elements as in the forward solver. Second, coarse level image of the optical coefficients are computed using data restricted to the coarser grid. Third, using coarse level optical coefficients as initial guess, finer level coefficients are computed at each subdomins. Local convergence of the inverse solver, when the variance of the optical coefficients are small, is proved using the abstract convergence theorem presented in. ${ }^{13}$ It is shown that the two level approach is indispensable in the algorithm due to the properties of local convergence result.

In summary, two domain decomposition technique are used in the present work. One is the twolevel overlapping domain decomposition method (Algorithm TMODDM) for forward solver and the other is two-level space decomposition method (Algorithm TMSDM) for inverse solver. We show that efficiency is gained using these two algorithms with respect to the overall computational complexity of the problem.

The paper is organized as follows: in the next section, forward solver based on photon diffusion equation model and minimization formulation of the inverse DOT is described. Two algorithms applying domain decomposition techniques to the forward and inverse steps of the minimization formulation is presented in Section 3. Finally in Section 4, we summarize the results of implementing these algorithms for a simulated problem.

\section{DIFFUSE OPTICAL TOMOGRAPHY}

\subsection{Photon Diffusion Equation in Frequency Domain}

Propagation of light in biological tissues $\Omega$ is described by photon diffusion equation in frequency domain derived from the simplest but non-trivial approximation in spherical harmonics of the Boltzmann equation.

$$
\begin{array}{rl}
-\nabla \cdot(\kappa(r) \nabla \Phi(r, \omega))+\mu_{a} \Phi(r, \omega)+\frac{i \omega}{c} \Phi(r, \omega)=q_{0}(r, \omega) & r \in \Omega \\
\Phi(r, \omega)+2 a \frac{\partial \Phi(r, \omega)}{\partial \nu}=0 & r \in \partial \Omega
\end{array}
$$

where $\Phi$ is the photon density, i.e. an integration of the number of photon per unit sphere, and $\mu_{a}, \mu_{s}^{\prime}, \kappa=\frac{1}{3\left(\mu_{a}+\mu_{s}^{\prime}\right)}$ are the absorption coefficient, the reduced scattering coefficient and the diffusion 
coefficient, respectively. The constant $a$, is related to the refraction on the boundary and $q_{0}$ is a source term. (2.1b) is called Robin boundary condition, which restricts the inward directed current to zero.

Usual assumption for the source term $q_{0}(r, \omega)=\delta\left(r-r_{0}\right)$ located at some point in $\bar{\Omega}$ is made. Consider $N_{s}$ sources and $N_{d}$ detectors at the boundary of $\Omega$. Let the photon density for each source term $q_{j}(r, \omega)=\delta\left(r-r_{j}\right), j=1, \cdots, N_{s}$ be $\Phi_{j}$. The measured boundary quantity is the normal component of the photon current, defined as the average number of photon in a given direction or simply $\frac{1}{2 a} \Phi_{j}$ due to Robin boundary condition (2.1b). Let detectors be $m_{i}, i=1, \cdots, N_{d}$. Then measured data at the boundary is described by $N_{s} * N_{d}$ column vector $\Phi_{i j}=\Phi_{j}\left(m_{i}\right)$ for $i j=$ $j+N_{d} *(i-1)$ for given $\kappa$ and $\mu_{a}$. Let measured data at $i$-th detector and $j$-th source be $\Gamma_{i j}$. Diffuse optical tomography is interpreted as finding the coefficients $\kappa$ and $\mu_{a}$ in some admissible class to minimize the square error between measured data $\Gamma_{i j}$ and the computed data $\Phi_{i j}\left[\kappa, \mu_{a}\right]$ attained by solving $(2.1)$.

\subsection{Forward solver using finite element method and adjoint method}

In this section, solution to forward problem is described for computing the boundary data and its Jacobian. Finite element method is used to solve for the photon densities and adjoint method is used to compute the Jacobian.

Consider the finite element space with basis functions $u_{i}^{\prime}, i^{\prime}=1, \cdots, N_{n}$ for some positive number $N_{n}$. Then the finite element formulation for (2.1) in this finite element space for $j$-th source $(j=$ $\left.1, \cdots, N_{s}\right)$ is as follows:

$$
\left[K(\kappa)+C\left(\mu_{a}\right)+1 /(2 a) A+i \omega / c B\right] \Phi_{j}=q_{j}
$$

where

$$
\begin{aligned}
K_{i^{\prime} j^{\prime}} & =\int_{\Omega} \kappa \nabla u_{i}^{\prime} \nabla u_{j}^{\prime} \\
C_{i^{\prime} j^{\prime}} & =\int_{\Omega} \mu_{a} u_{i}^{\prime} u_{j}^{\prime} \\
B_{i^{\prime} j^{\prime}} & =\int_{\Omega} u_{i}^{\prime} u_{j}^{\prime} \\
A_{i^{\prime} j^{\prime}} & =\int_{\partial \Omega} u_{i}^{\prime} u_{j}^{\prime}
\end{aligned}
$$

for $N_{n} \times N_{n}$ matrices $K, C, B, A$ and indices $i^{\prime}, j^{\prime}=1, \cdots, N_{n}$. By restricting $\Phi_{j}$ on detectors $m_{i}, i=$ $1, \cdots, N_{d}$, we obtain the boundary measurement data $\Gamma_{i j}$.

To implement the minimization procedure for optical coefficients, aside from computing $\Gamma_{i j}$ obtained from (2.2), the derivative of $\Gamma_{i j}$ with respect to the optical coefficients is needed. If we use Newton quotient for the computation of the derivative, computational burden increases proportional to the number of dimension of the space of optical coefficients. To reduce the computational burden, Jacobian is computed by the adjoint method using Born approximation. Born approximation is based on the assumption that the variance of optical coefficients and related photon densities are small as in. $^{22}$ This smallness assumption will be important factor in Section 3.2. Suppose piecewise linear finite element $P_{1}$ or piecewise bilinear finite element $Q_{1}$ is used. Suppose further that the diffusion and absorption coefficients are constant in each finite element. Let $N_{e}$ be the number of finite element in $\Omega$ and the derivative of $\Gamma_{i j}$ with respect to the coefficients be $J$. Then $J$ is $\left(N_{s} * N_{d}\right) \times\left(2 * N_{e}\right)$ matrix. For $j$-th source, $i$-th detector, and $k$-th element, $J$ is computed by

$$
\begin{aligned}
J(i j, k) & =1 /(2 a) \sum_{l, m=1}^{N n} \Phi_{l, i}^{*} V_{k}(l, m) \Phi_{m, j} \\
J\left(i j, N_{e}+k\right) & =1 /(2 a) \sum_{l, m=1}^{N n} \Phi_{l, i}^{*} V_{N e+k}(l, m) \Phi_{m, j}
\end{aligned}
$$


where

$$
\begin{aligned}
V_{k}(l, m) & =\int_{T_{k}} \nabla u_{l} \nabla u_{m} \\
V_{N_{e}+k}(l, m) & =\int_{T_{k}} u_{l} u_{m}
\end{aligned}
$$

for $k$-th element $T_{k}$.

\subsection{DOT as a nonlinear ill-posed optimization problem}

As stated in the introduction, DOT is a nonlinear ill-posed problem. Thus, regularization is needed to constrain and stabilize the solution. We chose Tikhonov type regularization. Newton-type, trust region method is used, which gives good results for large-scale constrained optimization problem of the type considered in this paper.

Given forward solver computing photon densities $\Gamma_{i j}$ at the boundary $\partial \Omega$ ( and its Jacobian $J_{i j, k}$ ), optical coefficients are estimated by the following inverse problem minimizing the error between measured boundary data and solution of the forward solver. Let $V$ be some admissible space of optical coefficients $\kappa$ and $\mu_{a}$.

$$
\min _{\kappa, \mu_{a} \in V} F\left(\kappa, \mu_{a}\right), \quad F\left(\kappa, \mu_{a}\right)=\frac{1}{2} \sum_{j=1}^{N_{s}} \sum_{i=1}^{N_{d}}\left(\frac{\Phi_{i, j}\left(\kappa, \mu_{a}\right)-\Gamma_{i, j}}{\sigma}\right)^{2}+\alpha \Upsilon\left(\kappa, \mu_{a}\right),
$$

where $\sigma$ is a standard deviation, $\alpha$ is the regularization parameter, and $\Upsilon$ is some measure in $V$. For the computational convenience assume $\sigma=1$ for the rest of this paper.

The unique identification of $\kappa$ and $\mu_{a}$ in (2.1) for infinite sources and infinite measurements when $\omega \neq 0$ can be easily shown by using the uniqueness results for isotropic case ${ }^{23}$ or for the case having anisotropic anomalies. ${ }^{24-26}$ But the uniqueness of $\kappa, \mu_{a}$ is not known generally for the finite sources and detectors. Thus the unique solvability of (2.4) is not assured leading to the ill-posedness of the DOT problem. But local uniqueness and convergence of (2.4) having positive Tikhonov regularization parameter is attained using local strong convexity of the operator $F$, which will be shown in (3.3).

The method of solving (2.4) is composed of two steps; the step to find the minimizing directions $\delta \kappa$ and $\delta \mu_{a}$ at the current coefficients $\kappa$ and $\mu_{a}$ and the step to perform line search on those minimizing directions. In the Newton-type method, minimizing direction is $-\left(F^{\prime \prime}\right)^{-1} F^{\prime}$, where $F^{\prime}$ and $F^{\prime \prime}$ are gradient and Hessian of $F$ with respect to $\kappa$ and $\mu_{a}$. One of efficient Newton-type method is a trust region method which computes the following minimization problem

$$
\min \left\{s \in W,\|D s\| \leq \Delta \mid \frac{1}{2} s^{t} F^{\prime \prime} s+s^{t} F^{\prime}\right\}
$$

where $D$ is scaling matrix, $\Delta$ is a trust region parameter, and $W$ is a subspace of $V$. To avoid heavy computation, two dimensional subspace composed of gradient direction and approximate Newton direction is chosen for subspace $W .{ }^{27}$

\section{DOMAIN DECOMPOSITION TECHNIQUES FOR DIFFUSE OPTICAL TOMOGRAPHY}

In this section, we describe the two domain decomposition methods considered in this paper, as applied to forward and inverse solutions. 


\subsection{Two level multiplicative overlapping domain decomposition method}

In forward solver of DOT formulation, we must find photon densities at detectors for all sources and compute its Jacobian with respect to the optical coefficients. Using adjoint method addressed in the previous section, computing Jacobian is straightforward by (2.3) given that photon densities for all sources and for all detectors (assuming detectors as virtual sources) are computed. A commonly used method for computing photon densities is via finite element method as in (2.2). In addition, we apply domain decomposition method to (2.2) in order to reduce the computation complexity of the problem.

The shape of photon density has a peak around the source and decrease rapidly far from the source. Thus one level multiplicative Schwarz method is not sufficient, since the domain far from the sources may neglect the existence of the sources, especially in the case where there are multiple subdomains. Hence we suggest two-level multiplicative overlapping domain decomposition method with following algorithm:

\section{Algorithm TMODDM}

1. Initialization Define subdomains, overlapping regions, maximum number of iterations.

2. Coarse grid correction Implement coarse grid correction using restriction and interpolation operators.

3. Subdomain correction Update photon densities for all sources at each subdomains. To use adjoint method, also compute photon densities for all detectors assuming detector as virtual source.

4. Stopping condition Iterate Coarse grid correction and Subdomain correction until maximum number of iteration is reached or the difference between the newly updated and previous photon densities in the overlapping region is sufficiently small.

5. Boundary measurement data Compute $\Gamma$ by restricting photon densities to the detectors on the boundary

6. Jacobian Compute Jacobian $J$ using adjoint method (2.3).

Suppose that $\Omega$ is a square in $\mathbb{R}^{n}, n=2,3$ discretized with $N^{n}$ mesh. Using a banded direct solver requires $O\left(N^{2 n}\right)$ floating points operations. But if two-level Schwarz method with $d$ decomposition of the domain is used, then the complexity is reduced to $O\left(\frac{N^{2 n}}{d}+N^{n}+d^{2}\right)$. If we use parallel computation with $d$ computers, each computer needs less than $O\left(\frac{N^{2 n}}{d^{2}}+N^{n}+d^{2}\right)$. Note that the number of iteration $M$ is bounded independent of $N^{n}, d$ and $N^{n} / d$ by the above result. Assuming $d<<N^{n}$, we can get approximately $\frac{M}{d}$ times $O\left(N^{2 n}\right)$ complexity for the present method in serial computing, and $\frac{M}{d^{2 n}}$ times $O\left(N^{2 n}\right)$ for the present method in parallel computing without considering data communication. By using multiple subdomains for the present method we achieve significant decrease in computational costs.

\subsection{Two level multiplicative space decomposition method}

We now describe the algorithm used in the inverse solver in detail. Assume $\kappa$ and $\mu_{a}$ are piecewise constant in each elements and let $\kappa$ and $\mu_{a}$ be some constant $\kappa_{l}$ and $\left(\mu_{a}\right)_{l}$ at $l$-th element. Take $V=\left\{x=\left(\kappa, \mu_{a}\right) \mid L \leq \kappa_{l},\left(\mu_{a}\right)_{l} \leq U\right\}$ and $\|x\|_{V}=\sqrt{\sum_{l=1}^{N e} \kappa_{l}^{2}+\left(\mu_{a}\right)_{l}^{2}}$ and $\Upsilon(x)=\frac{1}{2}\|x\|_{V}^{2}$. Then by simple computation,

$$
\Upsilon^{\prime}(x)=x, \quad \Upsilon^{\prime \prime}(x)=I_{2 N e} .
$$

where $I_{2 N e}$ is $2 N e \times 2 N e$ identity matrix. 
Let $b=\Phi\left(\kappa, \mu_{a}\right)-\Gamma$ and the Hessian of $b$ with respect to $\kappa, \mu_{a}$ be $H$, then $F^{\prime}$ and $F^{\prime \prime}$ are computed as follows:

$$
F^{\prime}(x)=J(x)^{t} b(x)+\alpha x, \quad F^{\prime \prime}(x)=J(x)^{t} J(x)+H(x) b(x)+\alpha I_{2 N e}
$$

Note that since Jacobian $J$, which is independent of $\delta x$ in (2.3) by Born approximation, is given the Hessian $H$ vanishes. Thus we get

$$
\left(\sigma_{2}+\alpha\right)\|y\|_{V}^{2} \leq y^{t} F^{\prime \prime}(x) y \leq\left(\sigma_{1}+\alpha\right)\|y\|_{V}^{2} \text { for } x, y \in V \text { and } x \text { is small. }
$$

for largest singular value $\sigma_{1}$ and smallest singular value $\sigma_{2}$ of $J$. If $N s * N d<2 N e, \sigma_{2}=0$. Even in the case that $N s * N d \geq 2 N e$ it is not clear that $\sigma_{2}>0$. However, with the aid of Tikhonov parameter $\alpha>0$, we can get strong convexity of $F$, from which follows that $C^{\prime}\|x\|_{V}^{2} \leq x^{t} F^{\prime \prime}(x) x$ for positive number $C^{\prime}$.

Convergence analysis for the space decomposition technique is based mostly on the strong convexity of objective function $F$. Which means that since in our case we derived the strong convexity result (3.3) from Born approximation, the convergence hold only if there is but a small variance in the optical coefficients $x$. Thus we need some modification on the previous approach to space decomposition technique. We need a good initial guess for the optical coefficients to be minimized. Incited by Algorithm TMODDM, coarse level optimized optical coefficients is used as an initial guess. As far as the authors' knowledge, how close these initial coarse level solutions are to the true finer level coefficients is not known. For the present work, we have assumed that this is sufficiently close to guarantee local convergence.

Let $\Omega$ be the sum of nonoverlapping $d$ subdomains $\Omega_{m}, m=1, \cdots, d$ and the number of finite elements in each subdomain $\Omega_{m}$ be $N e^{m}$. Then it is obvious that $N e=\sum_{m=1}^{d} N e^{d}$. The coarse level approximation of the coefficients are computed using the boundary data $\Gamma_{i j}^{c}$ on a coarse grid by using restriction operator to the measured data $\Gamma_{i j}$ used in Algorithm TMODDM.

\section{Algorithm TMSDM}

1. Initialization Choose subdomains, maximum number of iteration.

2. Restriction Restrict $\Gamma_{i j}$ to coarse level $\Omega$. Let $\Gamma_{i j}^{c}$ be this restriction boundary data.

3. Coarse level correction Find coefficients $x^{c}$ at this coarse level from $\Gamma_{i j}^{c}$ using Algorithm TMODDM.

4. Upsampling Compute upsampled data $x$ from $x^{c}$ at coarse level.

5. Subspace correction Using $x$ as an initial guess, update at all subdomains sequentially or parallel also using Algorithm TMODDM.

6. Downsampling If maximum number of iteration is reached or stopping condition is satisfied, stop. Otherwise, downsample $x$ to $x^{c}$ to coarse level and go to $\mathbf{3}$ using $x^{c}$ as an initial guess.

Roughly speaking, the computational advantage of space decomposition is as follows. Assume we use Newton direction for minimizing direction of (2.4) at each iterative step. Then we must compute $h$ satisfying

$$
\left(J^{t} J+\alpha I_{2 N e}\right) h=J^{t} b .
$$

(3.4) is solved by preconditioned conjugate gradient method and preconditioner is chosen by considering the structure of $J$. Assuming this preconditioned matrix computation is of order $O\left(N e^{4}\right)$ similar to band solver without space decomposition, the total computation for Algorithm TMSDM for $(3.4)$ is $O\left(d *\left(\frac{N e}{d}\right)^{4}+\left(\frac{N e}{2}\right)^{4}\right)=\left(\frac{1}{d^{3}}+\frac{1}{16}\right) O\left(N e^{4}\right)$ for serial computing and $\left(\frac{1}{d^{4}}+\frac{1}{16}\right) O\left(N e^{4}\right)$ for 
parallel computing. Thus the numerical efficiency is greatly increased for small increase in number of subdomains.

The local linear convergence of Algorithm TMSDM is shown below using Theorem 3.1. in. ${ }^{13}$ Theorem 1. Let $\kappa^{n}$ and $\mu_{a}^{n}$ are $n$-th step approximation for the Algorithm TMSDM and assume that $\kappa^{m+1}-\kappa^{m}$ and $\mu_{a}^{m+1}-\mu_{a}^{m}$ are sufficiently small for all $m=0, \cdots, n-1$. Then we have

$$
\left\|\left(\kappa^{n}, \mu_{a}^{n}\right)-\left(\kappa, \mu_{a}\right)\right\|_{V} \leq C_{1}\left(C_{2}\right)^{n}\left|F\left(\kappa^{0}, \mu_{a}^{0}\right)-F\left(\kappa, \mu_{a}\right)\right|
$$

where $C_{1}$ is a positive constant and $C_{2}$ is a positive constant less than 1.

Proof. Let $V=V_{1}+\cdots+V_{d}$, where $V_{l}, l=1, \cdots, d$ is the restriction of $V$ into $\Omega_{l}, l=1, \cdots, d$. Then, considering that $V$ is a convex closed subspace of reflexive Banach space $L^{2}(\Omega)$, we need only to show that assumptions $(3.1),(3,2)$ and $(3,3)$ for Theorem $3.1 \mathrm{in},{ }^{13}$ holds for our case to prove the above theorem. These assumption can easily be shown using the local strong convexity convexity (3.3), with the aid of Taylor expansion of $F^{\prime}$, mutual disjointness of $V_{l}, l=1, \cdots, d$, and Cauchy-Schwarz inequality.

\section{NUMERICAL TEST}

In this section, we will test the efficiency of proposed two algorithms: Algorithm TMODDM for forward solver and Algorithm TMSDM for inverse solver using simulated data.

\subsection{Algorithm TMODDM}

Refer to Figure 1 (b) for a picture of the simulated problem. Let $\Omega=[0,1] \times[0,1]$ be divided into $20 \times 20$ square elements. Let $\mu_{a}=1, \kappa=0.9$ and 0.1 at the white region and black region, respectively. Let the location of source be $(0.2,0)$.

In Figure 1, one level Schwarz method and two level Schwarz method is compared when $\Omega$ is divided into $2 \times 2$ square subdomains of the same size. Let the overlapping region be composed of all $2 w+1$ consecutive points on either side of the boundary points of each subdomain except $\partial \Omega$, where integer $w$ is the width of overlapping region. We have solved (2.2) by the proposed Algorithm TMODDM and one level Schwarz method. We compare the error of computed photon densities at each nodes between the two methods and LU method. The nodes are arranged as $(0,0),(1 / 20,0), \cdots,(1,0),(0,1 / 20), \cdots,(1,1)$. Thus the number of source point is 5 which has a apparent high peak in Figure 1. In Figure 1 (a),(c), and (e), the photon density approximation after 3, 6, 10 iterations of one-level Schwarz is plotted dashed line and the solution by LU method is plotted in solid line. In Figure 1 (d) and (f), the photon density approximation after 1,2 iterations by Algorithm TMODDM is plotted. Compared to one level Schwarz method, the discrimination of the solution of Algorithm TMODDM with that of LU method is nearly impossible in Figure 1(f). Note that the solution of LU method is exact, if we neglect round off error.

In Table 1, the effect of the number of subdomains and the width of overlapping region is considered. The $L^{2}$ error between LU method and Algorithm TMODDM is compared. As the width is larger, the error was decreased with a few exceptions. But the effect of the number of subdomains is not so remarkable. When iteration is small, the solution at 16 domains is more exact but the situation is reversed as iteration increases.

\subsection{Algorithm TMSDM}

In this section, various geometry of $\Omega, \kappa$, sources and detectors location, and the degree of noise and Tikhonov regularization parameter will be considered for the implementation of Algorithm TMSDM. The maximum number of iteration for coarse level and subspace correction for each subdomains are 10 . Let us denote as one cycle, this subspace correction after coarse level correction with 10 maximum iteration. As a forward solver we have used Algorithm TMODDM with maximum iteration 3 and overlapping width 1 . The decomposition of subdomains was chosen the same for the forward solver 


\begin{tabular}{c||cccccc}
\hline \hline Subdomains $\backslash$ Iteration & 1 & 2 & 3 & 4 & 5 & 6 \\
\hline \hline 4 domains, width 1 & $.964 \mathrm{e}-03$ & $.764 \mathrm{e}-07$ & $.191 \mathrm{e}-07$ & $.765 \mathrm{e}-09$ & $.755 \mathrm{e}-11$ & $.844 \mathrm{e}-12$ \\
\hline 4 domains, width 2 & $.834 \mathrm{e}-03$ & $.763 \mathrm{e}-07$ & $.160 \mathrm{e}-07$ & $.692 \mathrm{e}-09$ & $.110 \mathrm{e}-10$ & $.477 \mathrm{e}-12$ \\
\hline 4 domains, width 3 & $.717 \mathrm{e}-03$ & $.128 \mathrm{e}-06$ & $.151 \mathrm{e}-07$ & $.488 \mathrm{e}-09$ & $.159 \mathrm{e}-11$ & $.631 \mathrm{e}-12$ \\
\hline 16 domains, width 1 & $.119 \mathrm{e}-03$ & $.740 \mathrm{e}-07$ & $.191 \mathrm{e}-07$ & $.101 \mathrm{e}-08$ & $.193 \mathrm{e}-10$ & $.743 \mathrm{e}-12$ \\
\hline \hline
\end{tabular}

Table 1. The effect of multiple subdomains and overlapping width to the convergence.

and the inverse solver. In this section, we have fixed $\mu_{a}=1$ and considered only diffusion coefficient with two constants 0.9 (white region) and 0.1 (black region) as appears in Figure 2(a) and Figure $3(\mathrm{a})$. Although diffusion coefficient is used, it is a simple matter to consider absorption coefficient $\mu_{a}$ also.

In Figure 2, we have considered $\Omega=[0,1] \times[0,1]$ divided into $20 \times 20$. In Figure 2(a), the original image of diffusion coefficient is depicted. Sources and detectors are located all over the boundary as in Figure 2(b). The image is reconstructed using $2 \times 2$ subdomains after one cycle. The coarse level reconstruction and subspace correction at each subdomains, for case without noise, is shown in Figure 2(c) and (d). A $1 \%$ multiplicative noise was added to the measured data $\Gamma_{i j}, i=$ $1, \cdots, N d, j=1, \cdots, N s$ using Gaussian random noise generator with mean 0 and standard deviation 1. Reconstruction for the noisy data are shown in Figures 2(e) and 2(f). In Figure 2(e) and (f), the result of Algorithm TMSDM is compared between Tikhonov parameter $\alpha=0$ (no regularization) and $\alpha=1 . e-12$. The smallest singular value of Jacobian matrix was of order 1.e -13 . Thus the choice of regularization parameter plays a critical role in the reconstruction.

In Figure 3, we have considered thin slab type $\Omega=[0,1] \times[0,0.2]$ divided into $20 \times 4$ elements. Algorithm TMSDM with $4 \times 1$ subdomains is implemented to reconstruct $\kappa$ in Figure 3(a). The sources and detectors are located on all the nodes in the upper and lower part of $\partial \Omega$ as in Figure 3(b). $1 \%$ noise is added to photon densities at the boundary and we have used Tikhonov regularization value of 1.e - 13. After one cycle and two cycle, the approximated value is presented in Figure 3(c) and $3(\mathrm{~d})$.

\section{ACKNOWLEDGEMENT}

Various portions of this research were supported by the Center for Subsurface Sensing and Imaging Systems, under the Engineering Research Centers Program of the National Science Foundation (Award Number EEC-9986821), and Rensselaer Polytechnic Institute. This material is also based upon work supported by NSF-BES-0353160, ONR-N00014-04-1-0694, and US Army Medical ResearchW81XWH-04-1-0559. Any opinions, findings, and conclusions or recommendations expressed in this material are those of the authors and do not necessarily reflect the views of the National Science Foundation.

\section{REFERENCES}

1. D. A. Boas, D. H. Brooks, E. L. Miller, C. A. DiMarzio, M. Kilmer, R. J. Gaudette, and Q. Zhang, "Imaging the body with diffuse optical tomography," IEEE signal processing magazine, pp. 57$75,2001$.

2. X. Intes, C. Maloux, M. Guven, B. Yazici, and B. Chance, "Diffuse optical tomography with physiological and spatial a priori constraints," Phys. Med. Biol. 49, pp. N155-63, 2004.

3. M. Guven, B. Yazici, X. Intes, C. Maloux, and B. Chance, "Diffuse optical tomography with a priori anatomical information." submitted.

4. M. Dryja, "An additive Schwarz algorithm for two- and three- dimensional finite element elliptic problems," in Domain decomposition methods, pp. 168-72, SIAM, 1989. 
5. M. Dryja and O. Widlund, "Towards a unified theory of domain decomposition algorithms for elliptic problems," in Iterative methods for large systems, Academic press, 1990.

6. J. Bramble, J. Pasciak, J. Wang, and J. Xu, "Convergence estimates for product iterative methods with applications to domain decomposition," Math. Comp. 57, pp. 1-21, 1991.

7. P. L. Lions, "On the Schwarz alternating method I," in Proceedings of the first international symposium on domain decomposition methods for partial differential equations, pp. 2-42, SIAM, 1988.

8. P. L. Lions, "On the Schwarz alternating method II," in Domain decomposition methods, pp. 4770, SIAM, 1989.

9. P. L. Lions, "On the Schwarz alternating method III," in Proceedings of the third international symposium on domain decomposition methods for partial differential equations, pp. 202-23, SIAM, 1990.

10. I.-Y. Son, M. Guven, B. Yazici, and X. Intes, "A 2-level domain decomposition algorithm for inverse diffuse optical tomography," in Proceedings of ICIP, IEEE, 2004.

11. B. Smith, P. Bjorstad, and W. Gropp, Domain Decomposition, Cambridge University Press, 1996.

12. A. Quarteroni and A. Valli, Domain Decomposition Methods for Partial Differential Equations, Oxford Science Publications, 1999.

13. L. Badea, X.-C. Tai, and J. Wang, "Convergence rate analysis of a multiplicative Schwarz method for variational inequalities," SIAM J. Numer. Anal. 41, pp. 1052-73, 2003.

14. X.-C. Tai and P. Tseng, "Convergence rate analysis of an asynchronous space decomposition method for convex minimization," Mathematics of Computation 71, pp. 1105-35, 2001.

15. L. Badea and J. Wang, "An additive Schwarz method for variational inequalities," Mathematics of Computation 69, pp. 1341-54, 1999.

16. L. Badea, "On the Schwarz alternation method with more than two subdomains for nonlinear monotone problems," SIAM J. Numer. Anal. 28, pp. 179-204, 1991.

17. X.-C. Tai, "Rate of convergence for some constraint decomposition methods for nonlinear variational inequalities," Numer. Math. 93, pp. 755-86, 2003.

18. X.-C. Tai, "Some new domain decomposition and multigrid methods for variational inequalities," in Fourteenth International Conference on Domain Decomposition Methods, pp. 323-30, 2003 DDM.org, 2003.

19. J.-P. Zeng and S. Z. Zhou, "A domain decomposition method for a kind of optimization problems," Journal of Computational and Applied Mathematics 146, pp. 127-39, 2002.

20. X.-C. Tai, J. Froen, M. S. Espedal, and T. F. Chan, "Overlapping domain decomposition and multigrid methods for inverse problems," Contemporary Mathematics 218, pp. 523-29, 1998.

21. J. Xu, "Iterative methods by space decomposition and subspace correction," SIAM Reviews 34, pp. 581-613, 1992.

22. S. R. Arridge, "Optical tomography in medical imaging," Inverse Problems 15, pp. R41-93, 1999.

23. J. Sylvester and G. Uhlmann, "A global uniqueness theorem for an inverse boundary value problem," Ann. math. 125, pp. 153-69, 1987.

24. K. Kwon and D. Sheen, "Anisotropic inverse conductivity and scattering problems," Inverse Problems 18, pp. 745-56, 2002.

25. K. Kwon, "Identification of anisotropic anomalous region in inverse problems," Inverse Problems 20, pp. 1117-36, 2004.

26. V. Isakov, "On uniqueness in the inverse transmission scattering problem," Commun. Partial Diff. Eq. 15, pp. 1565-87, 1990.

27. R. H. Byrd, R. B. Schnabel, and G. A. Shultz, "Approximate solution of the trust region problem by minimization over two-dimensional subspaces," Mathematical programming 40, pp. 247-63, 1988. 


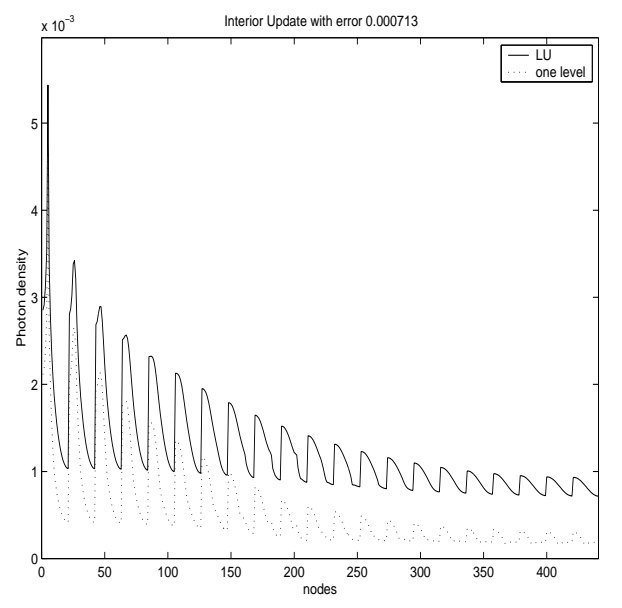

(a) One level Schwarz method, after 3 iterations

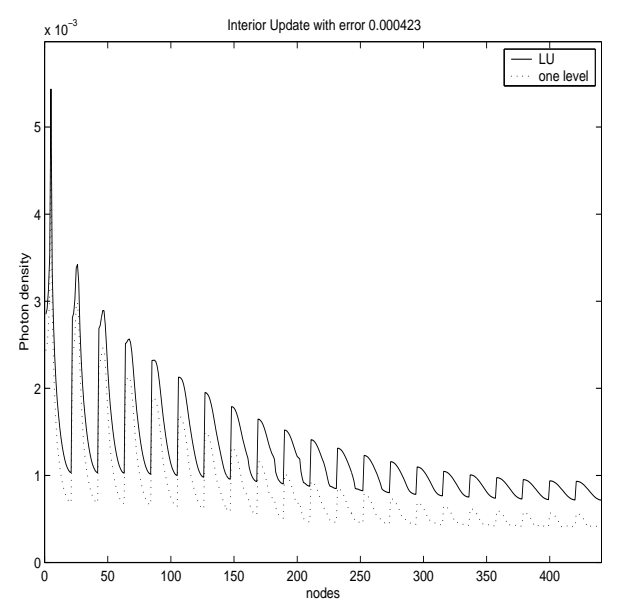

(c)One level Schwarz method, after 6 iterations

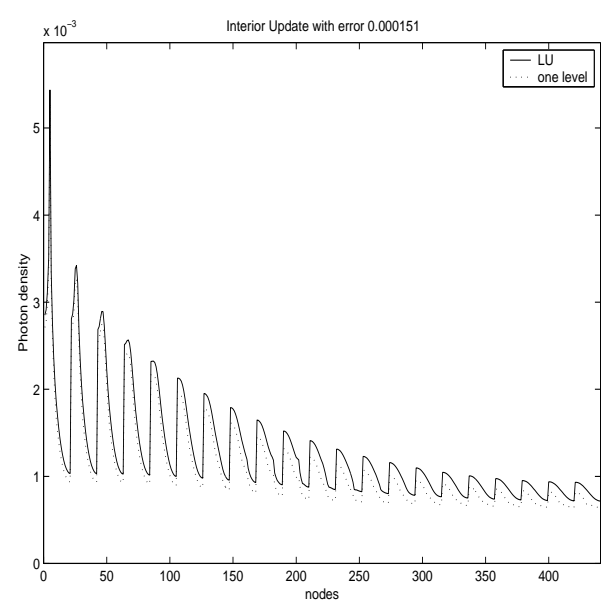

(e)One level Schwarz method, after 10 iterations

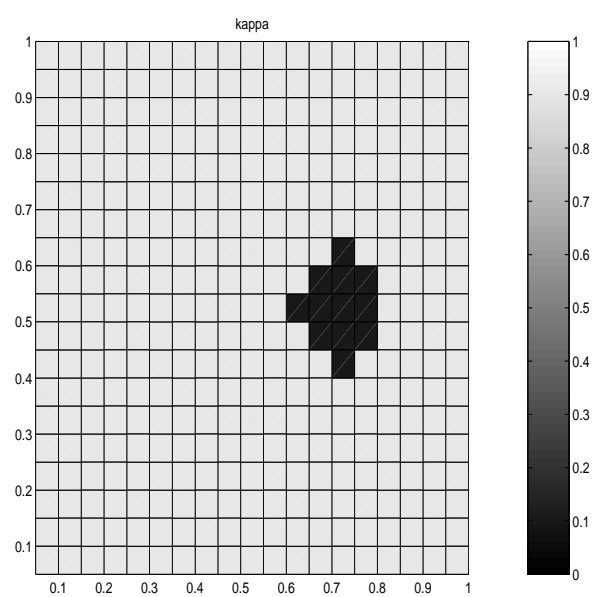

(b)The distribution of $\kappa$.

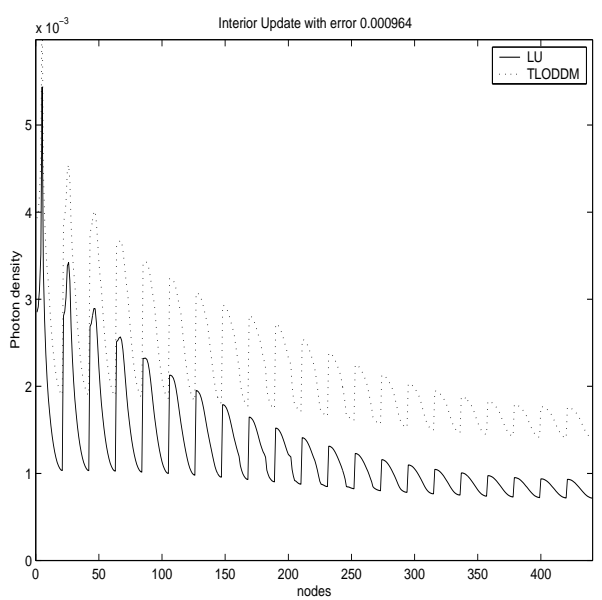

(d)Two level Schwarz method, after 1 iterations

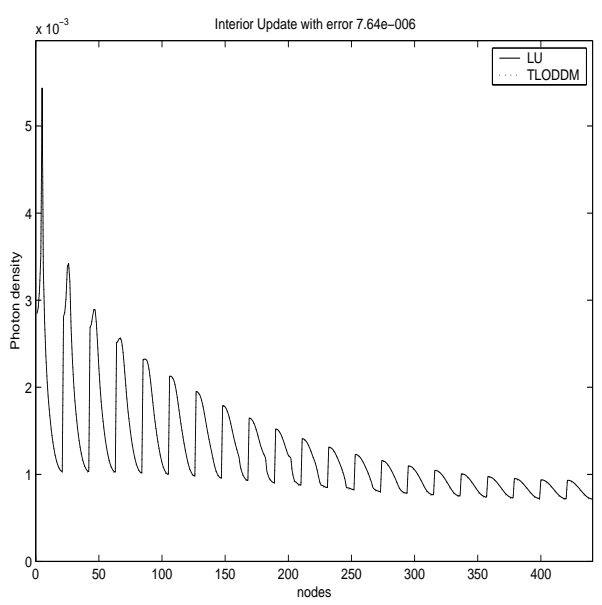

(f)Two level Schwarz method, after 2 iterations

Figure 1. Comparison between one level Schwarz method and two level Schwarz method 


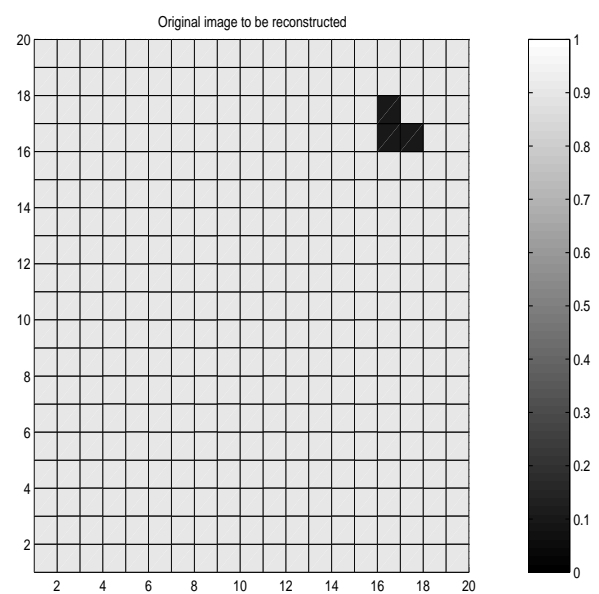

(a) Original image to be reconstructed.

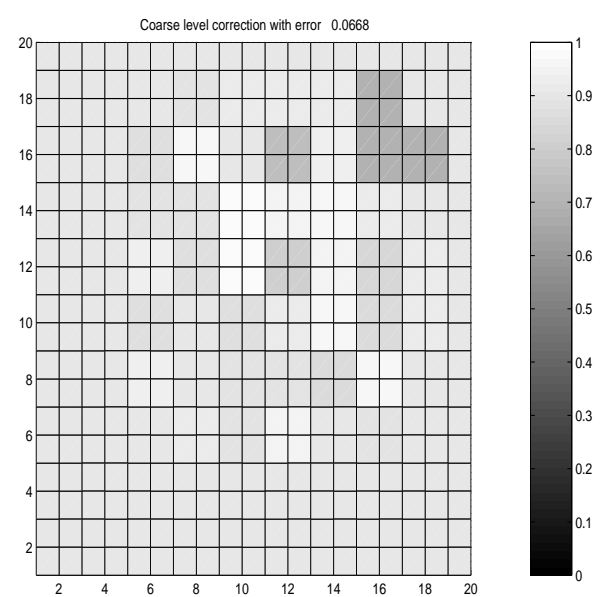

(c)Coarse level approximation, no noise

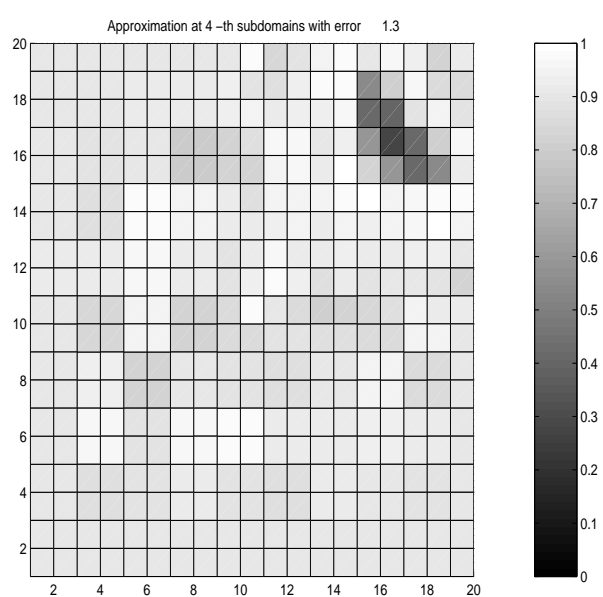

(e)Noise $1 \%$, Tikhonov parameter 0

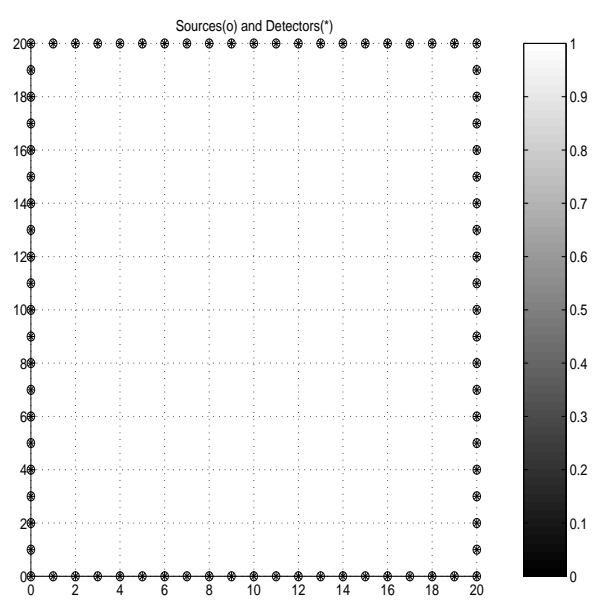

(b)The location of sources and detectors

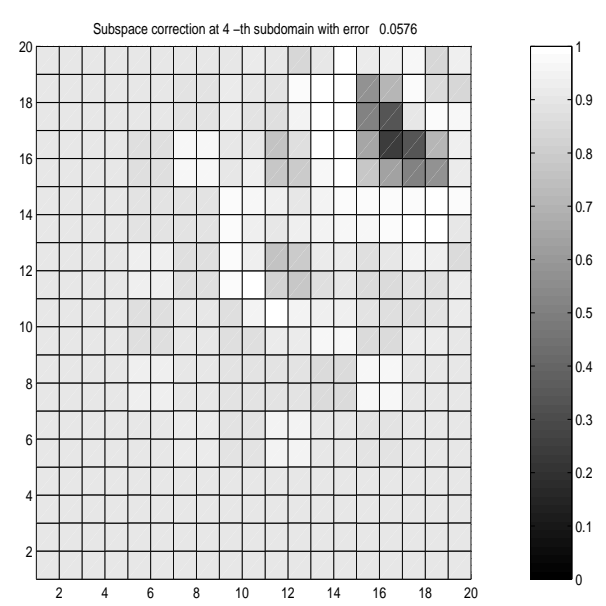

(d)Subspace correction at 4th subdomain, no noise

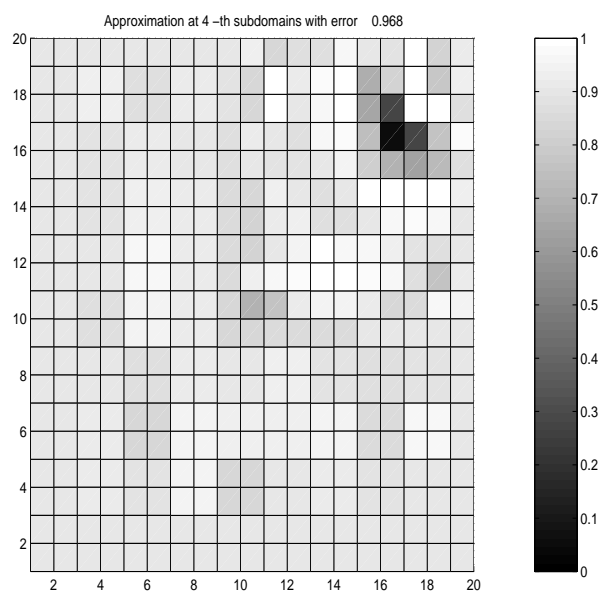

(f)Noise $1 \%$, Tikhonov parameter 1.e-12

Figure 2. Reconstruction using Algorithm TMSDM on squares with $2 \times 2$ subdomains 


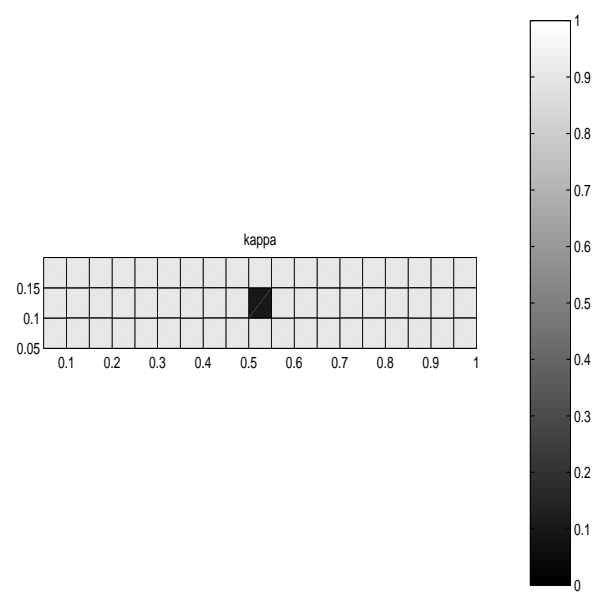

(a) Original image to be reconstructed.

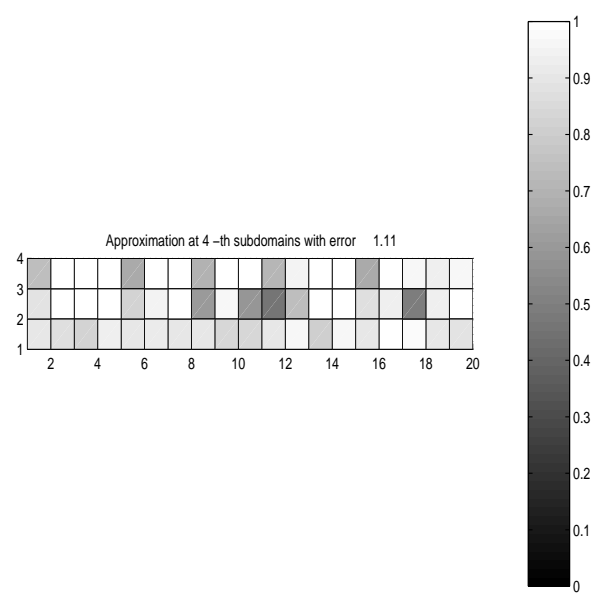

(c)After one cycle

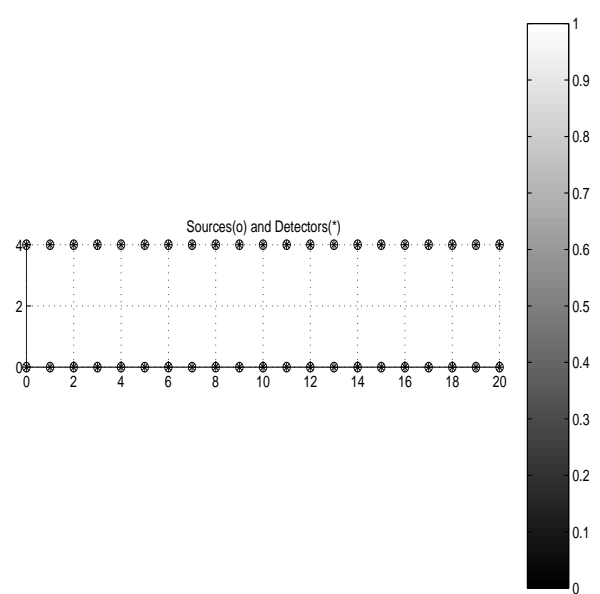

(b)Locations of sources and detectors

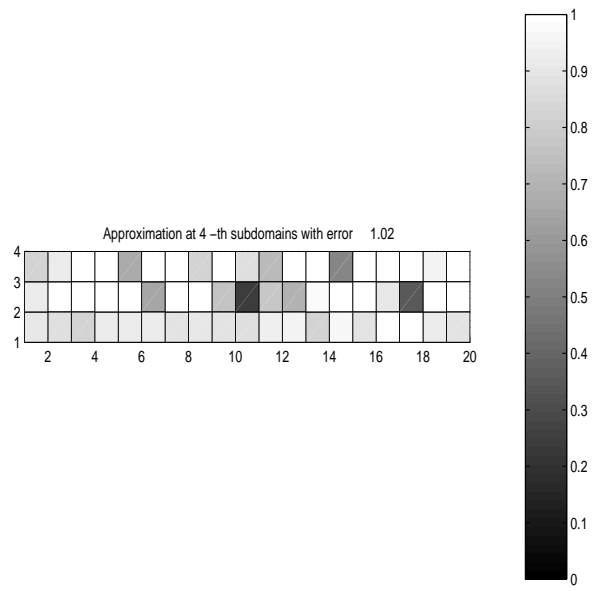

(d)After two cycles

Figure 3. Reconstruction using Algorithm TMSDM on thin slab with $4 \times 1$ subdomains and Tikhonov parameter $1 . e-13$ 